\title{
Distributional Response to Biases in Deterministic Superdiffusion
}

\author{
Takuma Akimoto* \\ Department of Mechanical Engineering, Keio University, Yokohama, 223-8522, Japan
}

(Received 26 December 2011; published 19 April 2012)

\begin{abstract}
We report on a novel response to biases in deterministic superdiffusion. For its reduced map, we show using infinite ergodic theory that the time-averaged velocity (TAV) is intrinsically random and its distribution obeys the generalized arcsine distribution. A distributional limit theorem indicates that the TAV response to a bias appears in the distribution, which is an example of what we term a distributional response induced by a bias. Although this response in single trajectories is intrinsically random, the ensemble-averaged TAV response is linear.
\end{abstract}

DOI: 10.1103/PhysRevLett.108.164101

PACS numbers: 05.45.Ac, 05.40.Fb, 87.15.Vv

Introduction.-Intrinsic randomness in macroscopic observables has been found in a broad range of processes more recently from biological transport to fluorescence of single nanocrystals [1-4]. Diffusion coefficients in biological transports show large fluctuations $[1,2]$. The ratio of an on-state in the fluorescence of nanocrystals does not converge to a constant and is different in each quantum dot [4]. The randomness of time-averaged observables can be characterized by power-law trapping time distributions in stochastic models, such as those in continuous time random walks (CTRWs) [5-7] and dichotomous stochastic processes [8]. Such randomness is due to the breakdown of the law of large numbers stemming from a diverging mean trapping time.

Dynamical systems with infinite-invariant measures can be viewed as stochastic processes generating random timeaveraged observables. Infinite ergodic theory plays an important role in elucidating such observables [9-14]. It guarantees that a time average of an observation function converges in distribution. In other words, time-averaged observables are intrinsically random if the invariant measure cannot be normalized. In dynamical systems generating subdiffusion, the distributional limit theorem for the diffusion coefficients obtained by the time-averaged mean square displacements (TAMSDs) has been shown using infinite ergodic theory [15].

In anomalous diffusion, the mean square displacement (MSD) grows nonlinearly with time, $\left\langle x(t)^{2}\right\rangle \propto t^{\alpha}(\alpha \neq 1)$. Diffusion is called subdiffusion if $\alpha<1$ and superdiffusion if $\alpha>1$. One mechanism generating subdiffusion is the divergence in the mean trapping time describing random walks like CTRW. If the mean trapping time diverges, the diffusion coefficients obtained from TAMSDs become random $[5-7,15]$. Three different mechanisms underlying

Published by the American Physical Society under the terms of the Creative Commons Attribution 3.0 License. Further distribution of this work must maintain attribution to the author(s) and the published article's title, journal citation, and DOI. superdiffusion have been identified: One stems from positive correlations in random walks, modeled by a fractional Brownian motion [16]; the second from persistent motions in random walks, called Lévy walks [17]; the third from very long jumps in random walks, called Lévy flights [18]. In Lévy walks and flights, the second moment of the length of persistence motion and of jumps diverges due to a power law. Such power laws are observed in Hamiltonian systems $[19,20]$, rotating flow [21], polymer diffusion [18], biological transport [22], intermittent search [23], and light diffusion [24]. Persistent times in Lévy walks and trapping times in CTRWs are characterized by indifferent fixed points in deterministic models [25]. Therefore, random transport coefficients will be observed in Lévy walk with the divergent mean persistent time.

Although random transport coefficients are universal in both unbiased and biased subdiffusions due to the divergent mean trapping time [26,27], it is not clear whether time-averaged observables are intrinsically random in superdiffusions. Moreover, little is known about responses of time-averaged drifts (TADs) to biases in superdiffusions whereas in anomalous diffusion a generalized Einstein relation holds [28]. In this Letter, we show a distributional limit theorem for TADs using deterministic superdiffusion models related to Lévy walks under bias and no bias. Surprisingly, a TAD is intrinsically random whether biased or unbiased. The result leads to a distributional response to bias; i.e., the response to bias would be characterized by a change in distribution.

Model.-Anomalous superdiffusion that originates from a persistent motion or a long jump has been studied for chaotic dynamical systems $[29,30]$. To study a response to a bias in deterministic superdiffusions related to Lévy walks, we propose an asymmetric deterministic diffusion model, constructed by introducing an asymmetry in the Geisel model [29]. In particular, we consider the following map $T: \mathbb{R} \rightarrow \mathbb{R}$,

$$
x_{n+1}=T\left(x_{n}\right),
$$

which have a translational symmetry $(L=\mathbb{Z})$ 


$$
T(x+L)=T(x)+L,
$$

and the map $T(x)$ is given by

$$
T(x)= \begin{cases}(x-L)+\left(\frac{x-L}{c}\right)^{z}+L-1, & x \in[L, L+c), \\ (x-L)-\left(\frac{L-x}{1-c}\right)^{z}+L+1, & x \in[L+c-1, L),\end{cases}
$$

where $c$ is a parameter characterizing an asymmetry. This model corresponds to a Lévy walk where leftward and rightward persistent time distributions have the same scaling exponent, i.e., $\psi(t) \propto t^{-z /(z-1)}$ but the probabilities of the leftward and rightward walk are different.

Reduced map.-As can be seen in Fig. 1, points near the fixed points, $x=0$ and $x=1$, on $[0,1]$ move to the left neighboring cell or the right neighboring cell, respectively. By translational symmetry, we can reduce an orbit of the map $T(x)$ to that of an intermittent map on [0,1] (Fig. 1). For example, we can obtain the following reduced map,

$$
R(x)=\left\{\begin{array}{lll}
x+\left(\frac{x}{c}\right)^{z}, & \bmod 1 & x \leq c, \\
x-\left(\frac{1-x}{1-c}\right)^{z}, & \bmod 1 & x>c,
\end{array}\right.
$$

with $0<c<1$. The invariant density of the reduced map is given by

$$
\rho(x)=h(x) x^{1-z}(1-x)^{1-z},
$$

where $h(x)$ is a continuous function satisfying $h(0) \neq 0$ and $h(1) \neq 0$ [31]. Thus, the invariant density cannot be normalized for $z \geq 2$. Consider the observation function

$$
f(x)= \begin{cases}-1, & x \in\left[0, c_{1}\right), \\ 0, & x \in\left[c_{1}, c_{2}\right), \\ +1, & x \in\left[c_{2}, 1\right),\end{cases}
$$

where $R\left(c_{1}\right)=1\left(c_{1}<c\right)$ and $R\left(c_{2}\right)=0\left(c_{2}>c\right)$. It follows that $X_{n}=f\left(x_{1}\right)+\ldots+f\left(x_{n}\right)$ is regarded as a one-dimensional random walk, where $x_{n}=R^{n}\left(x_{0}\right)$. The random walk $X_{n}$ corresponds to a Lévy walk where the
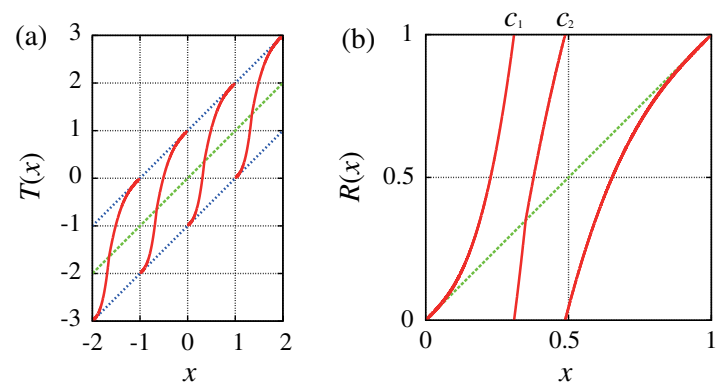

FIG. 1 (color online). (a) Asymmetric deterministic diffusion $T(x)(z=3.0$ and $c=0.35)$. (b) Its reduced map $R(x)$. persistent times distribution obeys a power law with exponent $\beta=z /(z-1)$.

Dependence of EAMSD on ensemble.-Ensembleaveraged MSD (EAMSD) and TAMSD are defined by

$$
\left\langle x_{m}^{2}\right\rangle=\lim _{K \rightarrow \infty} \frac{1}{K} \sum_{k=0}^{K-1}\left(T^{m}\left(x^{k}\right)-x^{k}\right)^{2},
$$

where $x^{k}$ is the $k$ th initial point, and

$$
\overline{\delta x_{m}^{2}}=\lim _{n \rightarrow \infty} \frac{1}{n} \sum_{k=0}^{n-1} f_{m}\left(x_{k}\right),
$$

where $f_{m}(x)=\left(T^{m}(x)-x\right)^{2}$, respectively. For a finite invariant measure $(z<2)$, ergodicity holds, i.e., EAMSD = TAMSD if initial points are distributed according to the invariant density of the reduced map. We note that ergodicity in an infinite measure space holds whereas ergodicity in the sense of time average being equal to the ensemble average does not hold for $z \geq 2$. When the invariant measure is infinite one, an equilibrium ensemble cannot be reproduced. The impossibility to reproduce an equilibrium ensemble leads to aging [32]. In unbiased cases $(c=0.5)$, EAMSD is studied by the renewal theory and continuous time random walk $[29,33]$ :

$$
\left\langle x_{m}^{2}\right\rangle_{E} \propto \begin{cases}m^{2}, & z \geq 2, \\ m^{3-1 /(z-1)}, & \frac{3}{2}<z<2, \\ m \ln m, & z=\frac{3}{2} \\ m, & 1<z<\frac{3}{2} .\end{cases}
$$

For $c<0.5$, drifting motion arises in a direction toward the right.

Unlike hyperbolic maps, statistical quantities determined by ensemble averages significantly depend on an initial ensemble in intermittent maps. In particular, it is shown that the behavior of the correlation function and the power spectrum density depend on an initial ensemble [34]. In renewal theory [35], there are two well-known processes, i.e., ordinary renewal and equilibrium renewal process. An initial ensemble corresponding to a specific renewal process is reproducible in dynamical systems. In particular, an initial ensemble for an equilibrium renewal process is an absolutely continuous invariant measure. Figure 2 shows that the EAMSD depends on an initial ensemble. If an initial ensemble of EAMSD has an invariant density (as in an equilibrium ensemble), all TAMSDs are equal to EAMSD (see Fig. 2).

Lamperti-Thaler's generalized arcsine law.-In dichotomous stochastic processes $\sigma_{n}$, an observable determined by the time average of an observation function $g\left(\sigma_{n}\right)$ is known to show random behavior if the mean residence time of a state diverges. In particular, the ratio of the occupation time of a state, $N_{n} / n$, does not converge to a constant, but converges in distribution, where $N_{n}$ is the occupation time of a state up to time $n$. The most classical 


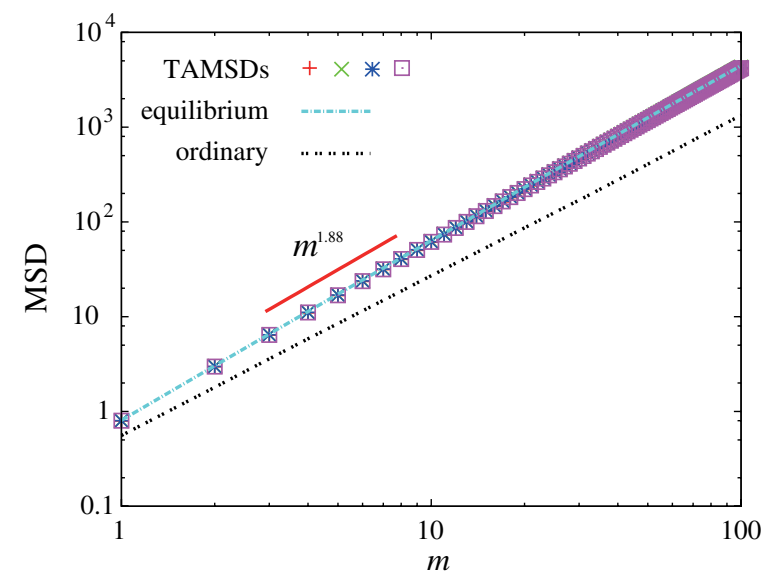

FIG. 2 (color online). Mean square displacements $(z=1.9$ and $c=0.5$ ). Different symbols are the TAMSDs calculated by different initial points. Dashed and dotted lines are the EAMSDs based on equilibrium and ordinary ensembles, respectively, where an ordinary ensemble is a uniform ensemble on $[0,1]$ and an equilibrium ensemble is the points after $10^{6}$ times iterations. The slope of the solid line is the theoretical scaling (9).

example is the arcsine law in coin tossing: the distribution of the ratio of the period that a player is on the positive side converges to the arcsine distribution [36]. Lamperti showed that the distribution of the ratio of the occupation time of a state converges to the generalized arcsine distribution in general dichotomous stochastic processes [37]. In Lévy walk, a position of a random walker at time $n$ is given by $x_{n}=2 N_{n}-n$, where $N_{n}$ is the occupation time of rightward walks up to time $n$. Therefore, the generalized arcsine distribution appears naturally in superdiffusions [38]. Moreover, the distribution becomes asymmetric if the mean of $N_{n} / n$ is not equal to $1 / 2$ [37]. In other words, a bias in superdiffusion generates such asymmetry.

In a dynamical system, the divergence of the mean residence time implies that the invariant density cannot be normalized [39]. Thaler has shown that the distribution of the time average of a characteristic function converges to the generalized arcsine distribution [10-12]. The generalized arcsine law is valid for the time average of a non- $L^{1}(\mu)$ function, i.e., $\int|g| d \mu=\infty$ [13]. LampertiThaler's generalized arcsine (LTGA) law [11] states that for a map $S:[0,1] \rightarrow[0,1]$ satisfying (i) $S([0, c])=[0,1]$ and $S([c, 1])=[0,1]$, (ii) $S^{\prime}(x)>1$ on $(0, c] \cup[c, 1)$; $S^{\prime}(0)=S^{\prime}(1)=1$, and (iii) $S(x)-x \sim a_{0} x^{p+1}(x \rightarrow 0)$ and $x-S(x) \sim a_{1}(1-x)^{p+1}(x \rightarrow 1)$ with $p>1$ and constants $a_{0}, a_{1}>0$, and $c \in(0,1)$, the time average of the $L_{\text {loc, } \mu}^{1}(0,1)$ function with finite mean [13] $g(x)$ converges in distribution:

$\operatorname{Pr}\left\{\frac{1}{n} \sum_{k=0}^{n-1} g \circ S^{k} \leq t\right\} \rightarrow \begin{cases}G_{\alpha, \beta}\left(\frac{t-b}{a-b}\right) & (a>b) \\ 1-G_{\alpha, \beta}\left(\frac{t-b}{a-b}\right) & (a<b),\end{cases}$ where $g(0)=a, g(1)=b$, and $\alpha=1 / p$,

$$
\beta=\frac{S^{\prime}\left(c_{+}\right)}{\left(a_{0} / a_{1}\right)^{1 / p} S^{\prime}\left(c_{-}\right)},
$$

and the probability density function (PDF) is given by

$$
G_{\alpha, \beta}^{\prime}(t)=\frac{\beta \sin \alpha}{\pi} \frac{t^{\alpha-1}(1-t)^{\alpha-1}}{\beta^{2} t^{2 \alpha}+2 \beta t^{\alpha}(1-t)^{\alpha} \cos \pi \alpha+(1-t)^{2 \alpha}} .
$$

The parameter $\beta$ characterizes an asymmetry of the PDF. This distribution is called the generalized arcsine distribution, which emerges in a subdiffusive transport [40] and weakly nonergodic statistical physics $[8,41]$. The mean of the occupation fraction of rightward walks $\left(x_{k}>c\right)$, $\left\langle N_{n} / n\right\rangle$, is given by $\left\langle N_{n} / n\right\rangle=1 /(1+\beta)$. Thus, $\beta \neq 1$ corresponds to a biased superdiffusion. We note that the exponents $\alpha$ and $\beta$ are determined by a behavior near the indifferent fixed points.

Distributional response to a bias._-LTGA law cannot be applied to the reduced map of the asymmetric deterministic diffusion model with $z \geq 2$ straightforwardly because the reduced map does not satisfy the condition (i). However, the condition (i) is not crucial because an important point in LTGA law is the reinjection to the indifferent fixed points. In fact, the conditions (i), (ii), and (iii) in [11] was generalized in [12]. The reinjection to the fixed point $x=0$ is determined by $\lim _{x \rightarrow c_{1}+0} R^{\prime}(x)$ and $\lim _{x \rightarrow c_{2}+0} R^{\prime}(x)$. Moreover, the reinjection to the fixed point $x=1$ is determined by $\lim _{x \rightarrow c_{1}-0} R^{\prime}(x)$ and $\lim _{x \rightarrow c_{2}-0} R^{\prime}(x)$. Because of $\lim _{x \rightarrow c_{1}+0} R^{\prime}(x)=\lim _{x \rightarrow c_{1}-0} R^{\prime}(x), \quad \lim _{x \rightarrow c_{2}+0} R^{\prime}(x)=$ $\lim _{x \rightarrow c_{2}-0} R^{\prime}(x)$, and $R^{\prime}(x)>1$ on $\left(0, c_{1}\right] \cup\left[c_{1}, c_{2}\right] \cup$ $\left[c_{2}, 1\right)$, the way of a reinjection to $x=0$ and $x=1$ is the same. Because the behavior near the indifferent fixed points is given by $S(x)-x \sim(x / c)^{z}(x \rightarrow 0)$ and $x-S(x) \sim$ $\{(1-x) /(1-c)\} z(x \rightarrow 1)$, we can apply LTGA law to the reduced map $R(x)$. Then, the exponents $\alpha$ and $\beta$ are given by

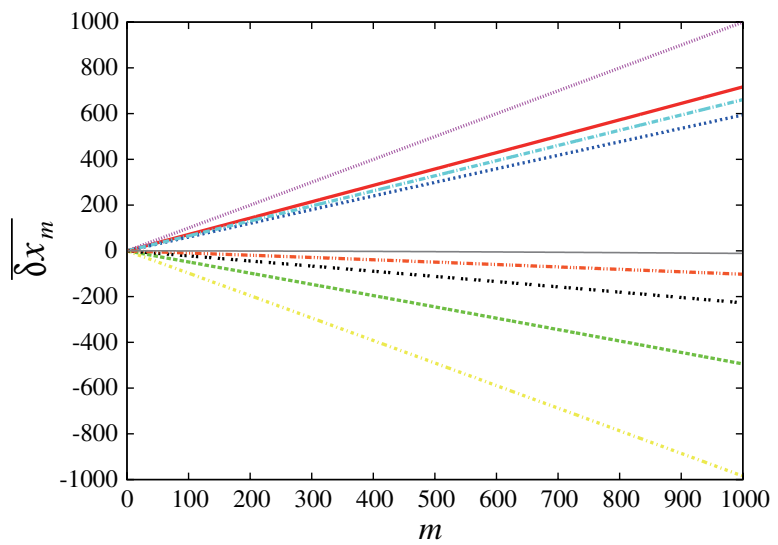

FIG. 3 (color online). Time-averaged drift $(z=3.0, c=0.45$, and $n=10^{5}$ ). TADs are calculated by different initial points. 


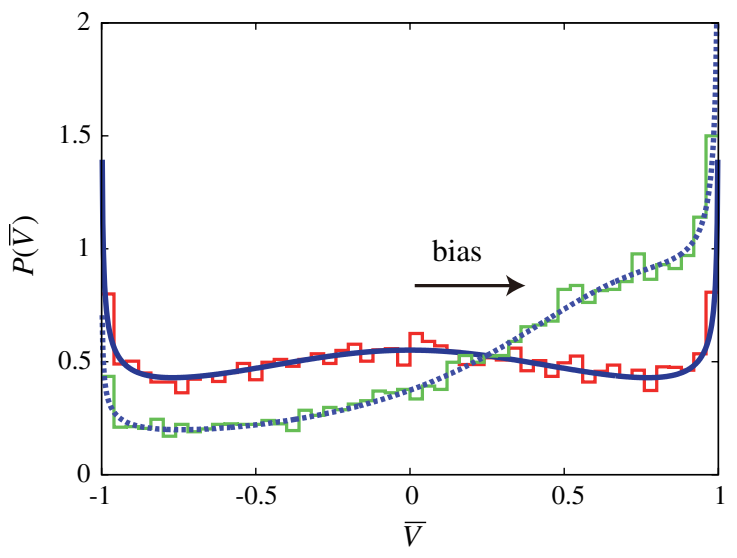

FIG. 4 (color online). Probability density function of $\bar{V}(z=$ 2.5 and $n=10^{7}$ ). Numerical results are represented by the solid histograms with the theoretical PDFs (15) for $c=0.5$ and $c=0.4$, respectively. If there is no bias $(c=0.5)$, the PDF is symmetric whereas it is not under bias.

$$
\alpha=\frac{1}{z-1} \quad \text { and } \quad \beta=\left(\frac{c}{1-c}\right)^{z / z-1} .
$$

First, we consider TAMSD, where the observation function $f_{m}(x)$ is the $L_{\text {loc, } \mu}^{1}(0,1)$ function with finite mean [13]. By LTGA law, $f_{m}(0)=m^{2}$ and $f_{m}(1)=m^{2}$, TAMSD converges to $m^{2}: \overline{\delta x_{m}^{2}}=m^{2}$ for all $c$ and $z \geq 2$. We note that the ballistic behavior, $\overline{\delta x_{m}^{2}}=m^{2}$, is not due to a drift for $c=0.5$. Next, we consider the time-averaged drift (TAD) defined by the time average of $v_{m}(x)=T^{m}(x)-x$ :

$$
\overline{\delta x_{m}}=\lim _{n \rightarrow \infty} \frac{1}{n} \sum_{k=0}^{n-1} v_{m}\left(x_{k}\right)
$$

which is also the $L_{\text {loc, } \mu}^{1}(0,1)$ function with finite mean. Figure 3 shows TADs obtained from different trajectories. By LTGA law, $v_{m}(0)=-m$ and $v_{m}(1)=m$, we have

$$
\operatorname{Pr}\left\{\overline{\delta x_{m}} / m \leq t\right\} \rightarrow 1-G_{\alpha, \beta}\left(\frac{t+1}{2}\right) .
$$

This distributional limit theorem states that the timeaveraged velocity, defined by $\bar{V} \equiv \overline{\delta x_{m}} / m$, under a bias is intrinsically random, i.e., distributional response. Numerical simulations are in good agreement with the theory (see Fig. 4). Because the ensemble average of $\bar{V}$ is given by $\langle\bar{V}\rangle=\left\langle\frac{2 N_{n}-n}{n}\right\rangle$, we have

$$
V \equiv\langle\bar{V}\rangle_{F}=\frac{1-\beta}{1+\beta},
$$

where $\langle\cdot\rangle_{F}$ is the ensemble average under an external bias. Figure 5 shows the response of $V$ to a bias $c$.

Generalized Einstein relation.-The asymmetric parameter $c$ with $0<c<1$ is considered to be the probability of leftward walk if a persistent motion is terminated. Let $c=e^{-(F / k T)} /\left(e^{-(F / k T)}+e^{F / k T}\right)$ be the leftward walk

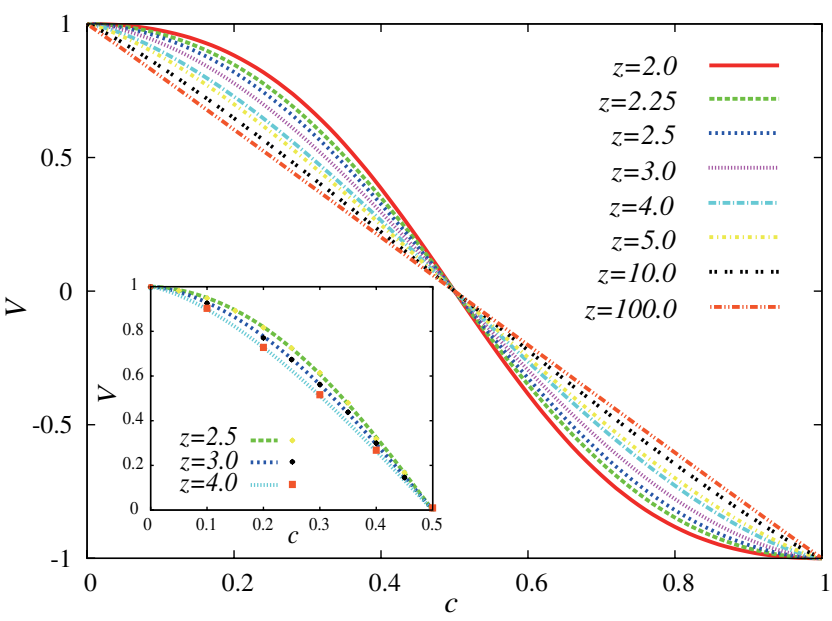

FIG. 5 (color online). Response to biases. Different curves are the response curves (16) for different $z$. Symbols are the results of numerical simulations in the inset figure.

probability, where $F$ is an external force, $T$ is a temperature, and $k$ is the Boltzmann constant [28]. We consider a small bias, $c \cong 1 / 2-F / k T$ for $F \rightarrow 0$. Expanding $\beta$ around $c=1 / 2$ and substituting it into $V$, we obtain the linear response of $V$ to a bias $F$ :

$$
V \sim 2 \frac{z}{z-1} \frac{F}{k T} .
$$

Although TADs are intrinsically random, the TAMSDs are not random and grow as $m^{2}$ for $z \geq 2$. Therefore, we have the following generalized Einstein relation for superdiffusion:

$$
\left\langle\overline{\delta x_{m}}\right\rangle_{F} \sim 2 \frac{z}{z-1} \frac{F}{k T} \sqrt{\overline{\delta x_{m}^{2}}}
$$

We note that TAMSD is calculated under no bias while the ensemble-averaged TAD is obtained under a bias.

Discussion. - We have found a distributional response in deterministic superdiffusion using the distributional limit theorem in infinite ergodic theory. The asymmetry parameter $c$ generates an asymmetry in the PDF of the timeaveraged velocity. In Lévy walk, an asymmetry property is affected by not only the probability of the jump direction but also the velocity. In a recent study we obtained a generalized Einstein relation for single trajectories using Hopf's ergodic theorem [27]. However, this theorem does not work in deterministic superdiffusion because the observation function $v_{m}(x)$ is not an $L^{1}(\mu)$ function. Therefore, a distributional response is essential in superdiffusion. Moreover, we noted that the generalized Einstein relation (18) is different from that in anomalous diffusion [28]. We hope that our finding, i.e., the distributional response, will be observed in experiments of random time-averaged observables.

The author thanks Tomoshige Miyaguchi for discussions. This work was partially supported by Grant-in-Aid for Young Scientists (B) (No. 22740262). 
*akimoto@z8.keio.jp

[1] I. Golding and E. C. Cox, Phys. Rev. Lett. 96, 098102 (2006).

[2] J. Szymanski and M. Weiss, Phys. Rev. Lett. 103, 038102 (2009).

[3] A. V. Weigel, B. Simonb, M. M. Tamkunc, and D. Krapf, Proc. Natl. Acad. Sci. U.S.A. 108, 6438 (2011).

[4] X. Brokmann et al., Phys. Rev. Lett. 90, 120601 (2003).

[5] A. Lubelski, I. M. Sokolov, and J. Klafter, Phys. Rev. Lett. 100, 250602 (2008).

[6] Y. He, S. Burov, R. Metzler, and E. Barkai, Phys. Rev. Lett. 101, 058101 (2008).

[7] T. Miyaguchi and T. Akimoto, Phys. Rev. E 83, 031926 (2011).

[8] G. Margolin and E. Barkai, J. Stat. Phys. 122, 137 (2006).

[9] J. Aaronson, An Introduction to Infinite Ergodic Theory (American Mathematical Society, Province, 1997).

[10] M. Thaler, Trans. Am. Math. Soc. 350, 4593 (1998).

[11] M. Thaler, Ergod. Theory Dyn. Syst. 22, 1289 (2002).

[12] M. Thaler and R. Zweimüller, Probab. Theory Relat. Fields 135, 15 (2006).

[13] T. Akimoto, J. Stat. Phys. 132, 171 (2008).

[14] N. Korabel and E. Barkai, Phys. Rev. Lett. 108, 060604 (2012).

[15] T. Akimoto and T. Miyaguchi, Phys. Rev. E 82, 030102(R) (2010).

[16] B. B. Mandelbrot and J. W. V. Ness, SIAM Rev. 10, 422 (1968).

[17] H. Scher and E.W. Montroll, Phys. Rev. B 12, 2455 (1975).

[18] A. Ott, J. P. Bouchaud, D. Langevin, and W. Urbach, Phys. Rev. Lett. 65, 2201 (1990).

[19] R. Venegeroles, Phys. Rev. Lett. 101, 054102 (2008).

[20] R. Venegeroles, Phys. Rev. Lett. 102, 064101 (2009).
[21] T.H. Solomon, E. R. Weeks, and H. L. Swinney, Phys. Rev. Lett. 71, 3975 (1993).

[22] L. Bruno, V. Levi, M. Brunstein, and M. A. Despósito, Phys. Rev. E 80, 011912 (2009).

[23] O. Bénichou, C. Loverdo, M. Moreau, and R. Voituriez, Rev. Mod. Phys. 83, 81 (2011).

[24] P. Barthelemy, J. Bertolotti, and D. S. Wiersma, Nature (London) 453, 495 (2008).

[25] G. Zumofen and J. Klafter, Phys. Rev. E 47, 851 (1993).

[26] I. M. Sokolov, E. Heinsalu, P. Hänggi, and I. Goychunk, Europhys. Lett. 86, 30009 (2009).

[27] T. Akimoto, Phys. Rev. E 85, 021110 (2012).

[28] J. Bouchaud and A. Georges, Phys. Rep. 195, 127 (1990).

[29] T. Geisel, J. Nierwetberg, and A. Zacherl, Phys. Rev. Lett. 54, 616 (1985).

[30] T. Miyaguchi, Prog. Theor. Phys. 115, 31 (2006).

[31] M. Thaler, Isr. J. Math. 46, 67 (1983).

[32] E. Barkai, Phys. Rev. Lett. 90, 104101 (2003).

[33] M. F. Shlesinger and J. Klafter, Phys. Rev. Lett. 54, 2551 (1985).

[34] T. Akimoto and Y. Aizawa, J. Korean Phys. Soc. 50, 254 (2007).

[35] D. R. Cox, Renewal Theory (Methuen, London, 1962).

[36] W. Feller, An Introduction to Probability Theory and its Applications (Wiley, New York, 1968), 3rd ed. Vol. 1.

[37] J. Lamperti, Trans. Am. Math. Soc. 88, 380 (1958).

[38] M. Bologna, G. Ascolani, and P. Grigolini, J. Math. Phys. (N.Y.) 51, 043303 (2010)

[39] T. Akimoto and Y. Aizawa, Chaos 20, 033110 (2010).

[40] M. A. Lomholt, I. M. Zaid, and R. Metzler, Phys. Rev. Lett. 98, 200603 (2007).

[41] A. Rebenshtok and E. Barkai, J. Stat. Phys. 133, 565 (2008). 